\title{
Residual Effect of Zn Fertilization on Wheat (Triticum aestivum L.) Grown in Soils with Divergent Characteristics
}

\author{
V. Girija Veni ${ }^{1 *}$, S. P. Datta ${ }^{2}$, R. K. Rattan ${ }^{2}$, M. C. Meena ${ }^{2}$ and K. L. Sharma ${ }^{1}$ \\ ${ }^{1}$ Central Research Institute for Dryland Agriculture, Santoshnagar, \\ Saidabad P.O., Hyderabad- 500059, India \\ ${ }^{2}$ Division of Soil Science and Agricultural Chemistry, Indian Agricultural Research Institute, \\ New Delhi-110012, India \\ *Corresponding author
}

\section{A B S T R A C T}

\begin{tabular}{|l|}
\hline K e y w o r d s \\
Grain Yield, Iron; \\
$\begin{array}{l}\text { Phosphorus, } \\
\text { Residual zinc, } \\
\text { Wheat, Zinc }\end{array}$ \\
\hline Article Info \\
\hline $\begin{array}{l}\text { Accepted: } \\
\text { 04 September } 2019 \\
\text { Available Online: } \\
\text { 10 October } 2019\end{array}$
\end{tabular}

Zinc deficiency is one of the causes for rheumatoid arthritis, diabetes, and cancers,

\section{Introduction}

Though one sixth of the global population resides in India, paradoxically, one third of about two billion people are suffering from vitamin and micronutrient deficiency in India due to heavy dependence on cereals grown in $\mathrm{Zn}$ - deficit soils. With the hypothesis that the residual $\mathrm{Zn}$ present in soil can also enhance the grain $\mathrm{Zn}$ content, a study was conducted with wheat variety 'HD 2329' to assess the grain concentrations of Zinc ( $\mathrm{Zn})$, Iron (Fe) and Phosphorus (P) grown on residual zinc in soils with divergent characteristics. Wheat crop was grown in the same pots on residual $\mathrm{Zn}$ fertility in which rice cultivars were grown with six $\mathrm{Zn}$ treatments in a completely randomised design (Factorial). The results of the study clearly indicated that wheat crop yield was not significantly influenced by the $\mathrm{Zn}$ applied to previous rice crop i.e. residual $\mathrm{Zn}$ fertility. However, the $\mathrm{Zn}$ concentration in wheat crop grown on residual fertility across the treatments ranged 29.6 to $33 \mathrm{mg} \mathrm{kg}^{-1}$. Residual $\mathrm{Zn}$ could maintain $11.5 \%$ more grain $\mathrm{Zn}$ concentration as compared to control. Among the $\mathrm{Zn}$ treatments, $5 \mathrm{mg} \mathrm{Zn} \mathrm{kg}{ }^{-1}$ (basal) plus FYM ( $5 \mathrm{mg} \mathrm{Zn} \mathrm{kg}^{-1}$ ) could maintain highest $\mathrm{Zn}\left(33 \mathrm{mg} \mathrm{kg}^{-1}\right)$ as compared to other treatments in wheat grain and straw. Among the soils, CRIDA soil could maintain higher grain $\mathrm{Zn}$ as compared to IARI and Karnal soil. There was an increase in DTPA-extractable $\mathrm{Zn}$ content in soils due to application of $\mathrm{Zn}$ in all the soils except in which $\mathrm{Zn}$ was supplied through foliar spray. Zinc application @ 5 $\mathrm{mg} \mathrm{Zn} \mathrm{kg}{ }^{-1}$ (basal) plus FYM (5 mg Zn kg${ }^{-1}$ ) significantly increased the DTPA- extractable $\mathrm{Zn}$ content over control in all the soils with mean value of $2.2 \mathrm{mg} \mathrm{kg}^{-1}$. More or less similar trend was observed in DTPA- extractable $\mathrm{Zn}$ in soil samples collected and analysed after the harvest of the wheat crop. which are associated with chronic
inflammation and oxidative stress (Kumar et
al., 2016). Zinc is involved in numerous
aspects of cellular metabolism. Zinc 
deficiency in humans is mainly due to heavy dependence on cereal food grains grown in Zn-deficit soils. And, among the cereals, next to rice, wheat is most important cereal crop. In India, rice-wheat cropping system contributes about $40 \%$ of the country's total food grain basket in an area of about $10.5 \mathrm{M}$ ha (Saharawat et al., 2010). In India, rice-wheat rotation is dominant in Punjab, Haryana, Bihar, Uttar Pradesh and Madhya Pradesh with a share of $75 \%$ of national food grain production (Mahajan and Gupta, 2009). Wheat grains contain about $25-30 \mathrm{mg} \mathrm{Zn} \mathrm{kg}^{-1}$ dry weight, while desired wheat grain $\mathrm{Zn}$ concentration should be $>50 \mathrm{mg} \mathrm{kg}^{-1}$ dry weight for a measurable impact of $\mathrm{Zn}$ biofortification on human health (Cakmak, 2008). In wheat seed, $\mathrm{Zn}$ is predominantly localized in the embryo and aleurone layer up to $150 \mathrm{mg}$ per $\mathrm{kg}$ seed whereas endosperm contains around $15 \mathrm{mg} \mathrm{Zn} \mathrm{per} \mathrm{kg}$ seed which is much less (Ozturk et al., 2006). Iron showed large variation among 66 wheat genotypes in a study carried in common wheat in central Asia, ranging from $25 \mathrm{mg} \mathrm{kg}^{-1}$ to 56 $\mathrm{mg} \mathrm{kg}^{-1}$ (mean $38 \mathrm{mg} \mathrm{kg}^{-1}$ ) and $\mathrm{Zn}$ concentration ranging between $20 \mathrm{mg} \mathrm{kg}^{-1}$ and $39 \mathrm{mg} \mathrm{kg}^{-1}$ (mean $28 \mathrm{mg} \mathrm{kg}^{-1}$ ) (Morgounov et al., 2007). A strong correlation between grain $\mathrm{Zn}$ and $\mathrm{Fe}$ concentrations was recorded in germplasm containing wild wheat (Cakmak et al., 2004). When 150 bread wheat lines were analysed, grain $\mathrm{Zn}$ ranged from 13.5 to 34.5 $\mathrm{mg} \mathrm{kg}^{-1}$; grain $\mathrm{Fe}$ content ranged from 28.9 to $50.8 \mathrm{mg} \mathrm{kg}^{-1}$ and found that durum, einkorn, emmer and spelt showed similar ranges as that of bread wheat (Zhao et al., 2009).

Wheat removes 47-91 g of $\mathrm{Zn}$ for every 3 tonnes of grains produced per hectare (Norton, 2012). Yet, farmers apply nitrogen, phosphorus and potassium fertilizers ignoring $\mathrm{Zn}$ application. Advantage of $\mathrm{Zn}$ application is $\mathrm{Zn}$ being highly immobile in soil, it has a residual effect for years on succeeding crops (Brennan, 2001; Rashid, 2005). Generally Zn application is recommended for every 3 years although the residual effect is dependent on soil types. It was reported that the residual effect of $\mathrm{Zn}$ last for 2 to 8 years (Lindsay, 1972). According to Brennan and Bolland (2007), this effect was estimated to be about 23 and 40 years for single application of 0.5 and $1 \mathrm{~kg} \mathrm{Zn} \mathrm{ha}{ }^{-1}$. Research findings report that residual effect of $\mathrm{Zn}$ can increase yield of subsequent crops. Residual effect of $10 \mathrm{~kg} \mathrm{Zn}$ $\mathrm{ha}^{-1}$ increased the grain yield in wheat (Khan et al., 2009) and increase was up to $30 \%$ over control (Khan et al., 2007). Increase in paddy yield up to $6.1 \%$ with residual application of 5 $\mathrm{kg} \mathrm{Zn} \mathrm{ha}{ }^{-1}$ was reported by Hussain (2004). There are also reports that residual effects of $\mathrm{Zn}$ increased the grain $\mathrm{Zn}$ content (Soleimani, 2012) and straw Zn content (Jat et al., 2012). Information on the residual effect of $\mathrm{Zn}$ fertilization after application is also desirable to determine the optimum application of $\mathrm{Zn}$ in crop rotation and to minimise the fertilizer usage. Most of the studies have focussed on studying the effect of residual $\mathrm{Zn}$ on yield on subsequent crops and there is very little information regarding its role in enriching the grain $\mathrm{Zn}$ content. Hence, our objective was to study residual effect of $\mathrm{Zn}$ application doses and methods followed in rice on the grain yield, grain $\mathrm{Zn}$ and $\mathrm{Fe}$ as well as $\mathrm{P}$ content in wheat.

\section{Materials and Methods}

Pot experiment was conducted to evaluate the residual effect of $\mathrm{Zn}$ (applied to the first croprice) on yield and nutrient content of wheat grown in a rice-wheat rotation at IARI, New Delhi. After the harvest of rice, the wheat (HD 2329) crop was grown only with basal dose of nitrogen, phosphorus and potassium but no $\mathrm{Zn}$ in the same layout. The first crop was grown in three different soils collected from Research Farm of IARI, New Delhi; farmer's field in Shamgarh, Karnal, Harayana and Hayathnagar Research Farm of Central Research Institute 
for Dry land Agriculture (CRIDA), Hyderabad with six treatments of $\mathrm{Zn}$ comprising control $\left(\mathrm{T}_{1}\right), 5 \mathrm{mg} \mathrm{Zn} \mathrm{kg}{ }^{-1}$ (basal) ( $\mathrm{T}_{2}$ ), foliar spray of $0.5 \% \mathrm{ZnSO}_{4}$ (bi-weekly) $\left(\mathrm{T}_{3}\right), 5 \mathrm{mg} \mathrm{Zn} \mathrm{kg}{ }^{-1}$ (basal) + foliar spray of $0.5 \% \mathrm{ZnSO}_{4}$ (biweekly) $\left(\mathrm{T}_{4}\right), 5 \mathrm{mg} \mathrm{Zn} \mathrm{kg}{ }^{-1}$ (basal) + FYM (5 $\left.\mathrm{mg} \mathrm{Zn} \mathrm{kg}{ }^{-1}\right)\left(\mathrm{T}_{5}\right), 2.5 \mathrm{mg} \mathrm{Zn} \mathrm{kg}{ }^{-1}$ (basal) + FYM (2.5 $\left.\mathrm{mg} \mathrm{Zn} \mathrm{kg}^{-1}\right)\left(\mathrm{T}_{6}\right)$ replicated two twice in a completely randomised design (Factorial). As per soil taxanomy, the IARI soil belongs to typic Haplustept, CRIDA soil to typic Haplustalf and Karnal soil Typic Ustochrepts subgroup. The IARI and CRIDA soils were having sandy clay loam texture while Karnal soil was having sandy clay texture. The experimental soils were divergent in their characteristics with varying $\mathrm{pH}, \mathrm{EC}$, mineral $\mathrm{N}$, available $\mathrm{P}, \mathrm{K}$ status and micronutrient content (Table 1).

At maturity, wheat crop was harvested manually. The grain and straw samples were washed under $0.01 \mathrm{~N} \mathrm{HCl}$ and under unning water to remove the adhering impurities.

The samples were air dried and placed in the oven at $65 \pm 5^{\circ} \mathrm{C}$. When the samples became brittle, they were hand pounded with pestle and motor to separate grain and husk and these samples were digested in diacid $\left(\mathrm{HNO}_{3}: \mathrm{HClO}_{4}\right)$ mixture. Iron and Zinc content in the digested samples were determined using Atomic Absorption spectrophotometer (Model : ZEEnit 700) and P content was estimated by vanado-molybdate- yellow colour method (Jackson 1973).Soil samples from each pot were collected at the harvest of wheat crop. The collected soil samples were air-dried and pulverized to pass through $2 \mathrm{~mm}$ sieve. Available Zn content was determined by Lindsay and Norvell (1978).

All the data obtained were statistically analyzed using the F-test as per the procedure given by Gomez and Gomez (1984). Least significant difference (LSD) values at $P=0.05$ were used to determine the significance of differences.

\section{Results and Discussion}

\section{Crop Performance}

After the harvest of the main crop of rice, the wheat crop was sown on residual $\mathrm{Zn}$ fertlity in same pots. When the wheat crop was grown on residual $\mathrm{Zn}$ fertility, wheat grain yield ranged from 8.9 to $9.3 \mathrm{~g} \mathrm{pot}^{-1}$ in IARI soil, 7.8 to $7.9 \mathrm{~g} \mathrm{pot}^{-1}$ in CRIDA soil and 8.2 to $8.5 \mathrm{~g}$ pot $^{-1}$ in Karnal soil (Table 2). In our study, no definite yield trend was observed. The Zn treatments did not have significant effect on wheat grain yield. Only soils had a significant effect on wheat grain yield. Similarly, the residual Zn had non-significant effect on straw yield of wheat. Straw yield ranged from 8.9 to $9 \mathrm{~g} \mathrm{pot}^{-1}$ in IARI soil, 7.9 to $8.9 \mathrm{~g} \mathrm{pot}^{-1}$ in CRIDA soil and 8.8 to $9.5 \mathrm{~g} \mathrm{pot}^{-1}$ in Karnal soil (Table 3). The two way interactive effect of soil and $\mathrm{Zn}$ treatment were found to be nonsignificant. In our study, the residual $\mathrm{Zn}$ did not have any significant effect on grain and straw yield in wheat. It was reported by Kulhare et al., (2014) that the residual effect of $2.5 \mathrm{t} \mathrm{FYM} \mathrm{ha}^{-1}$ was insufficient to increase the grain yield of wheat and the residual effect of $5 \mathrm{~kg} \mathrm{Zn} \mathrm{ha}{ }^{-1}$ was not significant for grain yield of wheat.

\section{Residual effect of $\mathrm{Zn}$ on grain and straw $Z n, F e$ and $P$ content in wheat}

The $\mathrm{Zn}$ content in wheat grain ranged 29.4 to $31.2 \mathrm{mg} \mathrm{kg}^{-1}$ grown on different soils with previous $\mathrm{Zn}$ application in rice crop and 35.8 to $36.5 \mathrm{mg} \mathrm{kg}^{-1}$ in wheat straw (Table 4). The soils had a significant effect in grain but not in straw. Among the $\mathrm{Zn}$ treatments, $5 \mathrm{mg} \mathrm{Zn} \mathrm{kg}{ }^{-1}$ (basal) plus FYM (5 mg Zn kg-1) could maintain highest $\mathrm{Zn} \quad\left(33 \quad \mathrm{mg} \quad \mathrm{kg}^{-1}\right)$ concentration as compared to other treatments in wheat grain and straw. Among the soils, 
CRIDA soil could maintain higher grain $\mathrm{Zn}$ as compared to IARI and Karnal soil. However, the two way interactive effects on grain and straw Zn content were found to be nonsignificant. Only soils had a significant effect on grain $\mathrm{Fe}$ content in wheat but not in straw. The grain Fe content in wheat ranged 29.9 to $30.3 \mathrm{mg} \mathrm{kg}^{-1}$ in IARI soil, 27 to $27.5 \mathrm{mg} \mathrm{kg}^{-1}$ in CRIDA soil and 28.6 to $29.6 \mathrm{mg} \mathrm{kg}^{-1}$ in Karnal soil and straw Fe ranged 193 to 196 $\mathrm{mg} \mathrm{kg}^{-1}$ in IARI soil, 194 to $197 \mathrm{mg} \mathrm{kg}^{-1}$ in CRIDA soil and 192 to $194 \mathrm{mg} \mathrm{kg}^{-1}$ in Karnal soil (Table 5). The two way interactive effects were found to be non-significant in both grain and straw. However, all main and interactive effects on $\mathrm{P}$ content in wheat grain and straw were non-significant (Table 6). The use efficiency of micronutrient is abysmally low hovering around two percent; it may go up to 5-6\% if residual effect has been taken (Rattan et al., 2008). This is reflected well in marginal enhancement of $\mathrm{Zn}$ content in wheat grown on residual fertility of $\mathrm{Zn}$ applied in previous crop (rice). Lyons et al., (2005) reported that the $\mathrm{Zn}$ content in wheat grain ranged 42 to 68 $\mathrm{mg} \mathrm{kg}^{-1}$ and $\mathrm{Zn}$ content of wheat grain in our study was considerably lower than those obtained by Lyons et al., (2005) and consistent with the findings of Cakmak et al., (2004) who reported that $\mathrm{Zn}$ content of wheat grain ranged from 8 to $34 \mathrm{mg} \mathrm{kg}^{-1}$ in the Middle Anatolia region. The wheat grain maintained $\mathrm{Fe}$ content similar to that of $\mathrm{Zn}$ content and the residual $\mathrm{Zn}$ did not interfere significantly on $\mathrm{Fe}$ accumulation in grain. It is reported by Cakmak et al., (1999) that soil application of $\mathrm{Zn}$ is most advisable to harness its residual effect by subsequent crops. Our study indicated that residual $\mathrm{Zn}$ also had a positive impact on grain $\mathrm{Zn}$ concentration. The residual $\mathrm{Zn}$ did not have any significant effect on grain $\mathrm{P}$ content although it has being reported that there exists antagonism between $\mathrm{P}$ and $\mathrm{Zn}$ (Hopkins and Elisworth, 2003; Hague et al., 2008). Earlier it was reported by Srinivasarao et al., (2008) that only at higher $\mathrm{P}$ levels, there exists an antagonistic interaction between $\mathrm{P}$ and $\mathrm{Zn}$. However, Soltangheisi et al., (2014) reported that $\mathrm{Zn}$ application did not have any effect on $\mathrm{P}$ concentration in corn.

\section{Residual effect of applied $\mathrm{Zn}$ on $\mathrm{Zn}$, Fe and $P$ uptake by wheat}

Zinc uptake by wheat ranged from 567 to 589 $\mu \mathrm{g}$ pot $^{-1}$, Fe uptake varied from 1928 to 1991 $\mu \mathrm{g} \operatorname{pot}^{-1}$ (Table 7) and $\mathrm{P}$ uptake varied from 36.3 to $37.1 \mathrm{mg} \operatorname{pot}^{-1}$ (Table 8) in wheat grown on different soils. The previously grown rice cultivars did not have a significant effect on $\mathrm{Zn}, \mathrm{Fe}$ and $\mathrm{P}$ uptake by wheat.

Among the different $\mathrm{Zn}$ treatments, $\mathrm{Zn}$ uptake was found highest in $5 \mathrm{mg} \mathrm{Zn} \mathrm{kg}^{-1}$ (basal) plus FYM (5 mg $\mathrm{Zn} \mathrm{kg}{ }^{-1}$ ). The $\mathrm{Zn}$ treatments (residual) could have a significant effect on $\mathrm{Zn}$ and non-significant effect on Fe and $\mathrm{P}$ uptake by wheat. The two way interactive effect of soil and treatment was found to be nonsignificant for $\mathrm{Zn}$ and $\mathrm{P}$ uptake and significant for Fe uptake. Individual effect of soils was found to be significant on $\mathrm{P}$ uptake. When simultaneous effect of treatments was studied, their two way interaction was found nonsignificant for $\mathrm{Zn}, \mathrm{Fe}$ and $\mathrm{P}$ uptake. Residual effect of $\mathrm{Zn}$ application through $\mathrm{ZnSO}_{4}$ and FYM could maintain higher $\mathrm{Zn}$ uptake by wheat as compared to other treatments. This finding is in line of earlier reports of Kulhare et al., (2014) where application of $\mathrm{Zn}$ along with FYM (residual) had positive impact on $\mathrm{Zn}$ uptake by wheat over control. Dhaliwal et al., (2014) reported that the $\mathrm{Zn}$ content in wheat grain ranged from 21.0 to $26.7 \mathrm{mg} \mathrm{kg}^{-1}$ and found significantly higher $\mathrm{Zn}$ uptake (102.7 to $117.3 \mathrm{~g} \mathrm{ha}^{-1}$ ) in the treatment that received organic manure @ $6 \mathrm{t} \mathrm{ha}{ }^{-1}$. This may be related to positive impact in terms of enhancing solubility of applied $\mathrm{Zn}$ in soil. Iron uptake by wheat was found higher in combined application of $\mathrm{Zn}$ and FYM. 
Table.1 Chemical characteristics of the experimental soils

\begin{tabular}{|c|c|c|c|}
\hline Parameter & IARI soil & CRIDA soil & Karnal soi \\
\hline pH & 8.2 & 6.0 & 8.4 \\
\hline $\operatorname{Ec}\left(\mathrm{dS} \mathrm{m}^{-1}\right)$ & 0.33 & 0.08 & 0.43 \\
\hline $\begin{array}{l}\text { Organic carbon ( } \mathrm{g} \mathrm{kg}^{-} \\
\text {1) }^{-}\end{array}$ & 3.42 & 1.49 & 3.48 \\
\hline $\begin{array}{lll}\text { Cation } & \text { exchange } \\
\text { capacity } & (c & \operatorname{mol}\left(\mathbf{p}^{+}\right) \\
\left.\text {kg }^{-1}\right) & & \end{array}$ & 14.3 & 11.5 & 19.5 \\
\hline Mineral N (Kg ha $\left.{ }^{-1}\right)$ & 15.7 & 14.3 & 21.9 \\
\hline Available $\mathrm{P}\left(\mathrm{Kg} \mathrm{ha}^{-1}\right)$ & 21.1 & 14.6 & 18.6 \\
\hline Available $\mathrm{K}\left(\mathrm{Kg} \mathrm{ha}^{-1}\right)$ & 279 & 149 & 273 \\
\hline Available $S\left(\mathrm{Kg} \mathrm{ha}^{-1}\right)$ & 22.3 & 18.4 & 28.7 \\
\hline \multicolumn{4}{|c|}{ DTPA Extractable micronutrients $\left(\mathrm{mg} \mathrm{kg}^{-1}\right)$} \\
\hline $\mathbf{Z n}$ & 2.16 & 0.78 & 1.32 \\
\hline $\mathbf{F e}$ & 14.9 & 7.21 & 23.2 \\
\hline $\mathbf{C u}$ & 1.15 & 0.69 & 0.79 \\
\hline Mn & 7.13 & 18.0 & 7.13 \\
\hline
\end{tabular}

Table.2 Residual effect of $\mathrm{Zn}$ on grain yield $\left(\mathrm{g} \mathrm{pot}^{-1}\right)$ of wheat grown on three different soil types

\begin{tabular}{|c|c|c|c|c|}
\hline \multirow[t]{2}{*}{ Zinc treatment $(\mathbf{T})$} & \multicolumn{4}{|c|}{ Soil (S) } \\
\hline & IARI & CRIDA & KARNAL & Mean \\
\hline Control & 9.0 & 7.5 & 8.5 & 8.3 \\
\hline $5 \mathrm{mg} \mathrm{Zn} \mathrm{kg}^{-1}$ (basal) & 9.2 & 7.8 & 8.5 & 8.5 \\
\hline $\begin{array}{l}\text { Foliar spray of } 0.5 \% \mathrm{ZnSO}_{4} \\
\text { (bi-weekly) }\end{array}$ & 8.9 & 8.1 & 8.4 & 8.4 \\
\hline $\begin{array}{l}5 \mathrm{mg} \mathrm{Zn} \mathrm{kg}{ }^{-1}(\text { basal) }+ \text { foliar } \\
\text { spray of } 0.5 \% \mathrm{ZnSO}_{4} \text { (bi- } \\
\text { weekly) }\end{array}$ & 9.3 & 7.8 & 8.7 & 8.6 \\
\hline $\begin{array}{l}5 \mathrm{mg} \mathrm{Zn} \mathrm{kg}^{-1} \text { (basal) + FYM } \\
\left(5 \mathrm{mg} \mathrm{Zn} \mathrm{kg}^{-1}\right)\end{array}$ & 9.4 & 7.4 & 8.2 & 8.3 \\
\hline $\begin{array}{l}2.5 \mathrm{mg} \mathrm{Zn} \mathrm{kg}^{-1} \text { (basal) + FYM } \\
\left(2.5 \mathrm{mg} \mathrm{Zn} \mathrm{kg}^{-1}\right)\end{array}$ & 9.1 & 8.5 & 7.8 & 8.5 \\
\hline CD (P=0.05) & $S=0.3$ & & $=\mathbf{N S}$ & $\mathrm{S} \times \mathrm{T}=\mathrm{NS}$ \\
\hline
\end{tabular}


Table.3 Effect of $\mathrm{Zn}$ treatment $(\mathrm{T})$ on straw yield $\left(\mathrm{g} \mathrm{pot}^{-1}\right)$ of wheat grown on three different soil types

\begin{tabular}{|c|c|c|c|c|}
\hline \multirow[t]{2}{*}{ Zinc treatment $(\mathrm{T})$} & \multicolumn{4}{|c|}{ Soil (S) } \\
\hline & IARI & CRIDA & KARNAL & Mean \\
\hline Control & 8.8 & 7.2 & 9.6 & 8.5 \\
\hline $5 \mathrm{mg} \mathrm{Zn} \mathrm{kg}^{-1}$ (basal) & 8.9 & 8.2 & 9.3 & 8.8 \\
\hline $\begin{array}{l}\text { Foliar spray of } 0.5 \% \mathrm{ZnSO}_{4} \\
\text { (bi-weekly) }\end{array}$ & 8.6 & 7.7 & 9.1 & 8.4 \\
\hline 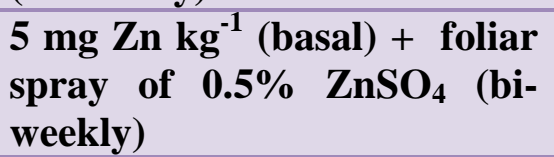 & 9.1 & 7.9 & 9.7 & 8.9 \\
\hline $\begin{array}{l}5 \mathrm{mg} \mathrm{Zn} \mathrm{kg}^{-1} \text { (basal) + FYM } \\
\left(5 \mathrm{mg} \mathrm{Zn} \mathrm{kg}^{-1}\right)\end{array}$ & 9.1 & 8.6 & 8.9 & 8.8 \\
\hline $\begin{array}{l}2.5 \mathrm{mg} \mathrm{Zn} \mathrm{kg-1} \text { (basal) + FYM } \\
(2.5 \mathrm{mg} \mathrm{Zn} \mathrm{kg})\end{array}$ & 9.3 & 10.2 & 7.5 & 9.0 \\
\hline $\mathrm{CD}(\mathbf{P}=0.05)$ & & $T=\mathbf{N S}$ & $\mathrm{Sx}_{\mathrm{x}}$ & \\
\hline
\end{tabular}

Table.4 Effect of residual $\mathrm{Zn}$ on $\mathrm{Zn}$ content $\left(\mathrm{mg} \mathrm{kg}^{-1}\right)$ in wheat grown on three different soil types

\begin{tabular}{|c|c|c|c|c|c|c|c|c|}
\hline \multirow{3}{*}{ Zinc treatment $(\mathbf{T})$} & \multicolumn{8}{|c|}{ Soil (S) } \\
\hline & \multicolumn{2}{|l|}{ IARI } & \multicolumn{2}{|c|}{ CRIDA } & \multicolumn{2}{|c|}{ KARNAL } & \multicolumn{2}{|l|}{ Mean } \\
\hline & Grain & Straw & Grain & Straw & Grain & Straw & Grain & Straw \\
\hline Control & 30.2 & 27.9 & 30.2 & 29.7 & 28.5 & 23.4 & 29.6 & 26.9 \\
\hline $5 \mathrm{mg} \mathrm{Zn} \mathrm{kg}^{-1}$ (basal) & 30.6 & 34.9 & 32.0 & 33.9 & 29.1 & 35.6 & 30.6 & 34.8 \\
\hline $\begin{array}{l}\text { Foliar spray of } 0.5 \% \\
\mathrm{ZnSO}_{4} \text { (bi-weekly) }\end{array}$ & 30.3 & 31.3 & 30.5 & 30.0 & 28.7 & 31.0 & 29.9 & 30.4 \\
\hline $\begin{array}{l}5 \mathrm{mg} \mathrm{Zn} \mathrm{kg}^{-1} \text { (basal) + } \\
\text { foliar spray of } 0.5 \% \\
\mathrm{ZnSO}_{4} \text { (bi-weekly) }\end{array}$ & 31.2 & 38.9 & 31.7 & 40.8 & 29.2 & 38.4 & 30.7 & 39.4 \\
\hline $\begin{array}{l}5 \mathrm{mg} \mathrm{Zn} \mathrm{kg}{ }^{-1}(\text { basal })+ \\
\text { FYM }\left(5 \mathrm{mg} \mathrm{Zn} \mathrm{kg}^{-1}\right)\end{array}$ & 32.9 & 44.6 & 35.6 & 44.4 & 30.5 & 43.4 & 33.0 & 44.1 \\
\hline $\begin{array}{l}2.5 \mathrm{mg} \mathrm{Zn} \mathrm{kg-1} \text { (basal) } \\
+ \text { FYM (2.5 mg Zn kg } \\
\left.\text { 1 }^{-}\right)\end{array}$ & 32.3 & 42.5 & 33.9 & 41.1 & 29.8 & 38.8 & 32.0 & 40.8 \\
\hline $\mathrm{CD}(\mathrm{P}=\mathbf{0 . 0 5})$ & $\begin{array}{l}\text { Grain } \\
\text { Straw }\end{array}$ & & $\begin{array}{l}S=1 . \\
S=N\end{array}$ & & $\begin{array}{l}\mathbf{T}=\mathbf{1} \\
\mathbf{T}=\mathbf{3}\end{array}$ & & $\begin{array}{l}S \times T \\
S \times T\end{array}$ & \\
\hline
\end{tabular}


Table.5 Effect of residual $\mathrm{Zn}$ on Fe content $\left(\mathrm{mg} \mathrm{kg}^{-1}\right)$ in wheat grown on three different soil types

\begin{tabular}{|c|c|c|c|c|c|c|c|c|}
\hline \multirow[t]{3}{*}{ Zinc treatment $(\mathbf{T})$} & \multicolumn{8}{|c|}{ Soil (S) } \\
\hline & \multicolumn{2}{|l|}{ IARI } & \multicolumn{2}{|c|}{ CRIDA } & \multicolumn{2}{|c|}{ KARNAL } & \multicolumn{2}{|l|}{ Mean } \\
\hline & Grain & Straw & Grain & Straw & Grain & Straw & Grain & Straw \\
\hline Control & 32.1 & 194 & 25.9 & 197 & 30.8 & 193 & 29.6 & 195 \\
\hline $5 \mathrm{mg} \mathrm{Zn} \mathrm{kg}^{-1}$ (basal) & 28.5 & 198 & 26.8 & 199 & 29.0 & 194 & 28.1 & 197 \\
\hline $\begin{array}{l}\text { Foliar spray of } 0.5 \% \\
\mathrm{ZnSO}_{4} \text { (bi-weekly) }\end{array}$ & 30.7 & 193 & 27.3 & 198 & 30.2 & 193 & 29.4 & 195 \\
\hline $\begin{array}{l}5 \mathrm{mg} \mathrm{Zn} \mathrm{kg}{ }^{-1} \text { (basal) } \\
+\quad \text { foliar spray of } \\
0.5 \% \mathrm{ZnSO}_{4} \text { (bi- } \\
\text { weekly) }\end{array}$ & 28.6 & 196 & 26.9 & 195 & 28.2 & 194 & 27.9 & 195 \\
\hline $\begin{array}{l}5 \mathrm{mg} \mathrm{Zn} \mathrm{kg}^{-1} \text { (basal) } \\
+ \text { FYM (5 mg Zn kg- } \\
\text { 1) }\end{array}$ & 30.8 & 192 & 28.8 & 191 & 28.7 & 190 & 29.4 & 191 \\
\hline $\begin{array}{l}2.5 \mathrm{mg} \underset{\mathrm{Zn}}{\mathrm{kg}^{-1}} \\
(\text { basal) }+ \text { FYM } \\
\text { mg Zn kg }^{-1} \text { ) }\end{array}$ & 30.3 & 192 & 27.8 & 194 & 27.9 & 192 & 28.7 & 193 \\
\hline $\mathrm{CD}(\mathrm{P}=\mathbf{0 . 0 5})$ & $\begin{array}{l}\text { Grai } \\
\text { Stra }\end{array}$ & & $\begin{array}{l}\mathbf{S}=1 \\
\mathbf{S}=1\end{array}$ & & $\begin{array}{l}T= \\
T=\end{array}$ & & $\begin{array}{l}\mathbf{S} \mathbf{x} \\
\mathbf{S} \mathbf{x}\end{array}$ & $\begin{array}{l}=\mathbf{N S} \\
=\mathbf{N S}\end{array}$ \\
\hline
\end{tabular}

Table.6 Effect of $\mathrm{Zn}$ application on $\mathrm{P}$ content $\left(\mathrm{mg} \mathrm{g}^{-1}\right)$ in wheat grown as influenced by different soil types

\begin{tabular}{|c|c|c|c|c|c|c|c|c|}
\hline \multirow[t]{3}{*}{ Zinc treatment $(\mathbf{T})$} & \multicolumn{8}{|c|}{ Soil (S) } \\
\hline & \multicolumn{2}{|l|}{ IARI } & \multicolumn{2}{|c|}{ CRIDA } & \multicolumn{2}{|c|}{ KARNAL } & \multicolumn{2}{|l|}{ Mean } \\
\hline & Grain & Straw & Grain & Straw & Grain & Straw & Grain & Straw \\
\hline Control & 3.37 & 0.97 & 3.37 & 0.97 & 3.55 & 1.03 & 3.43 & 0.99 \\
\hline $5 \mathrm{mg} \mathrm{Zn} \mathrm{kg}^{-1}$ (basal) & 3.30 & 0.90 & 3.23 & 0.87 & 3.45 & 1.05 & 3.34 & 0.94 \\
\hline $\begin{array}{l}\text { Foliar spray of } 0.5 \% \\
\mathrm{ZnSO}_{4} \text { (bi-weekly) }\end{array}$ & 3.28 & 0.88 & 3.30 & 0.90 & 3.38 & 0.95 & 3.32 & 0.91 \\
\hline $\begin{array}{l}5 \mathrm{mg} \mathrm{Zn} \mathrm{kg}^{-1} \text { (basal) + } \\
\text { foliar spray of } 0.5 \% \\
\mathrm{ZnSO}_{4} \text { (bi-weekly) }\end{array}$ & 3.38 & 0.98 & 3.38 & 0.98 & 3.30 & 0.90 & 3.36 & 0.96 \\
\hline $\begin{array}{l}5 \mathrm{mg} \mathrm{Zn} \mathrm{kg}{ }^{-1} \text { (basal) + } \\
\left.\text { FYM (5 mg Zn kg } \text { }^{-1}\right)\end{array}$ & 3.32 & 0.90 & 3.30 & 0.90 & 3.50 & 1.05 & 3.37 & 0.95 \\
\hline $\begin{array}{l}2.5 \mathrm{mg} \mathrm{Zn} \mathrm{kg}^{-1} \text { (basal) + } \\
\text { FYM }\left(2.5 \mathrm{mg} \mathrm{Zn} \mathrm{kg}^{-1}\right)\end{array}$ & 3.30 & 0.90 & 3.33 & 0.93 & 3.30 & 0.90 & 3.31 & 0.91 \\
\hline CD $(P=0.05)$ & $\begin{array}{l}\text { Grain } \\
\text { Straw }\end{array}$ & & $\begin{array}{l}=\mathbf{N S} \\
=\mathrm{NS}\end{array}$ & & & $\begin{array}{l}\mathbf{S} \\
\mathbf{S}\end{array}$ & $\begin{array}{l}=\mathbf{N S} \\
=\mathbf{N S}\end{array}$ & \\
\hline
\end{tabular}


Table.7 Effect of $\mathrm{Zn}$ application on $\mathrm{Zn}$ and Fe uptake $\left(\mu \mathrm{g} \mathrm{pot}^{-1}\right)$ by wheat as influenced by previous grown rice cultivars

\begin{tabular}{|c|c|c|c|c|c|c|c|c|}
\hline \multirow[t]{3}{*}{ Zinc treatment $(\mathbf{T})$} & \multicolumn{8}{|c|}{ Soil (S) } \\
\hline & \multicolumn{2}{|c|}{ IARI } & \multicolumn{2}{|c|}{ CRIDA } & \multicolumn{2}{|c|}{ KARNAL } & \multicolumn{2}{|c|}{ Mean } \\
\hline & $\mathrm{Zn}$ & $\mathrm{Fe}$ & $\mathrm{Zn}$ & $\mathrm{Fe}$ & $\mathrm{Zn}$ & $\mathrm{Fe}$ & $\mathrm{Zn}$ & $\mathrm{Fe}$ \\
\hline Control & 517 & 2006 & 440 & 1612 & 472 & 2131 & 477 & 1917 \\
\hline $5 \mathrm{mg} \mathrm{Zn} \mathrm{kg}^{-1}$ (basal) & 595 & 2038 & 526 & 1855 & 579 & 2061 & 567 & 1984 \\
\hline $\begin{array}{l}\text { Foliar spray of } 0.5 \% \\
\mathrm{ZnSO}_{4} \text { (bi-weekly) }\end{array}$ & 541 & 1941 & 467 & 1756 & 522 & 2009 & 510 & 1902 \\
\hline $\begin{array}{l}5 \mathrm{mg} \mathrm{Zn} \mathrm{kg}{ }^{-1} \text { (basal) + } \\
\text { foliar spray of } 0.5 \% \\
\mathrm{ZnSO}_{4} \text { (bi-weekly) }\end{array}$ & 641 & 2046 & 569 & 1776 & 632 & 2140 & 614 & 1988 \\
\hline 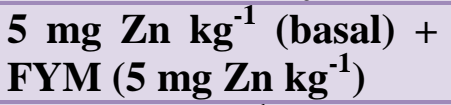 & 717 & 2046 & 645 & 1887 & 636 & 1922 & 666 & 1952 \\
\hline $\begin{array}{l}2.5 \mathrm{mg} \mathrm{Zn} \mathrm{kg}^{-1}(\text { basal) + } \\
\text { FYM }\left(2.5 \mathrm{mg} \mathrm{Zn} \mathrm{kg}^{-1}\right)\end{array}$ & 689 & 2077 & 704 & 2221 & 527 & 1673 & 640 & 1991 \\
\hline $\mathrm{CD}(\mathrm{P}=\mathbf{0 . 0 5})$ & $\begin{array}{l}\mathrm{Zn} \\
\mathrm{Fe}\end{array}$ & & $\begin{array}{l}=38.3 \\
139\end{array}$ & & $\begin{array}{l}54.3 \\
\text { NS }\end{array}$ & & & \\
\hline
\end{tabular}

Table.8 Effect of $\mathrm{Zn}$ application on $\mathrm{P}$ uptake $\left(\mathrm{mg} \mathrm{pot}^{-1}\right)$ by wheat as influenced by previous grown rice cultivars

\begin{tabular}{|c|c|c|c|c|}
\hline \multirow[t]{2}{*}{ Zinc treatment $(\mathrm{T})$} & \multicolumn{4}{|c|}{ Soil (S) } \\
\hline & IARI & CRIDA & KARNAL & Mean \\
\hline Control & 38.8 & 32.2 & 40.0 & 37.0 \\
\hline $5 \mathrm{mg} \mathrm{Zn} \mathrm{kg}^{-1}$ (basal) & 38.3 & 32.6 & 39.2 & 36.7 \\
\hline $\begin{array}{l}\text { Foliar spray of } 0.5 \% \\
\mathrm{ZnSO}_{4} \text { (bi-weekly) }\end{array}$ & 36.9 & 33.6 & 37.0 & 35.9 \\
\hline $\begin{array}{l}5 \mathrm{mg} \mathrm{Zn} \mathrm{kg}{ }^{-1} \text { (basal) + } \\
\text { foliar spray of } 0.5 \% \\
\mathrm{ZnSO}_{4} \text { (bi-weekly) }\end{array}$ & 40.2 & 34.3 & 37.7 & 37.4 \\
\hline $\begin{array}{l}5 \mathrm{mg} \mathrm{Zn} \mathrm{kg-1} \text { (basal) + } \\
\text { FYM }\left(5 \mathrm{mg} \mathrm{Zn} \mathrm{kg}^{-1}\right)\end{array}$ & 39.3 & 32.3 & 37.9 & 36.5 \\
\hline $\begin{array}{l}2.5 \mathrm{mg} \mathrm{Zn} \mathrm{kg}{ }^{-1}(\text { basal })+ \\
\text { FYM }\left(2.5 \mathrm{mg} \mathrm{Zn} \mathrm{kg}^{-1}\right)\end{array}$ & 38.5 & 37.7 & 32.9 & 36.4 \\
\hline \multicolumn{2}{|l|}{$\mathrm{CD}(\mathrm{P}=\mathbf{0 . 0 5})$} & $S=2.1$ & \multicolumn{2}{|c|}{$S \times T=N S$} \\
\hline
\end{tabular}


Table.9 DTPA extractable $\mathrm{Zn}\left(\mathrm{mg} \mathrm{kg}^{-1}\right)$ in three different soil types as influenced by residual $\mathrm{Zn}$ application at the harvest of wheat

\begin{tabular}{|c|c|c|c|c|}
\hline \multirow[t]{2}{*}{ Zinc treatment (T) } & \multicolumn{4}{|c|}{ Soil (S) } \\
\hline & IARI & CRIDA & KARNAL & Mean \\
\hline Control & 1.53 & 0.74 & 1.07 & 1.11 \\
\hline $5 \mathrm{mg} \mathrm{Zn} \mathrm{kg}^{-1}$ (basal) & 1.86 & 1.13 & 1.23 & 1.41 \\
\hline $\begin{array}{l}\text { Foliar spray of } 0.5 \% \mathrm{ZnSO}_{4} \\
\text { (bi-weekly) }\end{array}$ & 1.74 & 1.15 & 1.11 & 1.33 \\
\hline $\begin{array}{l}5 \mathrm{mg} \mathrm{Zn} \mathrm{kg}{ }^{-1} \text { (basal) + foliar } \\
\text { spray of } 0.5 \% \mathrm{ZnSO}_{4} \text { (bi- } \\
\text { weekly) }\end{array}$ & 2.06 & 1.25 & 1.14 & 1.48 \\
\hline $\begin{array}{l}5 \mathrm{mg} \mathrm{Zn} \mathrm{kg} \\
\left(5 \mathrm{mg} \mathrm{Zn} \mathrm{kg}^{-1}\right)\end{array}$ & 2.12 & 1.46 & 1.33 & 1.64 \\
\hline $\begin{array}{l}2.5 \mathrm{mg} \mathrm{Zn} \mathrm{kg}^{-1} \text { (basal) + FYM } \\
\left(2.5 \mathrm{mg} \mathrm{Zn} \mathrm{kg}^{-1}\right)\end{array}$ & 1.99 & 1.07 & 1.19 & 1.42 \\
\hline \multicolumn{2}{|l|}{ CD $(P=0.05) \quad S=0.1$} & \multicolumn{2}{|c|}{$\mathbf{T}=\mathbf{0 . 2}$} & $\Gamma=\mathrm{NS}$ \\
\hline
\end{tabular}

Fig.1 Mean DTPA extractable zinc content in soils after the harvest of first crop (rice) and second crop (wheat)

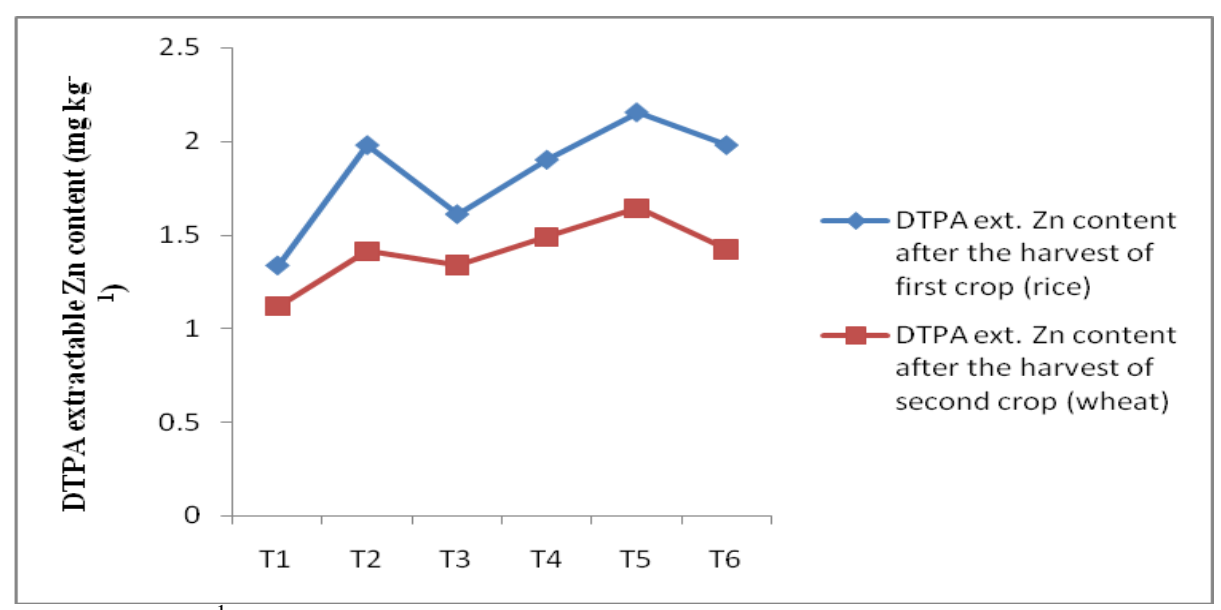

$\mathrm{T}_{1}=$ Control; $\mathrm{T}_{2}=5 \mathrm{mg} \mathrm{Zn} \mathrm{kg}{ }^{-1}$ (basal); $\mathrm{T}_{3}=$ Foliar spray of $0.5 \% \mathrm{ZnSO}_{4}$ (bi-weekly); $\mathrm{T}_{4}=5 \mathrm{mg} \mathrm{Zn} \mathrm{kg}{ }^{-1}$ (basal) + foliar spray of $0.5 \% \mathrm{ZnSO}_{4}$ (bi-weekly); $\mathrm{T}_{5}=5 \mathrm{mg} \mathrm{Zn} \mathrm{kg}{ }^{-1}$ (basal) + FYM (5 mg Zn kg$) ; \mathrm{T}_{6}=2.5 \mathrm{mg} \mathrm{Zn} \mathrm{kg}$ (basal) $^{-1}$ + FYM $\left(2.5 \mathrm{mg} \mathrm{Zn} \mathrm{kg}^{-1}\right)$ 
Phosphorus uptake by wheat remained unaffected by $\mathrm{Zn}$ application. Phosphorus uptake by wheat was not significantly affected by $\mathrm{Zn}$ application. Zhu et al., (2001) also reported that the $\mathrm{Zn}$ application had little effect on tissue $\mathrm{P}$ concentration and $\mathrm{P}$ uptake.

\section{DTPA-extractable $\mathrm{Zn}$ in post harvest soil of} wheat

The DTPA-extractable $\mathrm{Zn}$ after the harvest of wheat crop was found high in IARI soil (1.88 $\left.\mathrm{mg} \mathrm{kg}^{-1}\right)$ followed by Karnal soil (1.19 $\mathrm{mg} \mathrm{kg}^{-}$ ${ }^{1}$ ) and was low in CRIDA soil (1.14 $\left.\mathrm{mg} \mathrm{kg}^{-1}\right)$. The soils imparted a significant effect on DTPA-extractable $\mathrm{Zn}$. The $\mathrm{Zn}$ treatments applied to previous rice had a significant effect on DTPA-extractable Zn. Among the different treatments, the DTPA-extractable Zn was found highest in $5 \mathrm{mg} \mathrm{Zn} \mathrm{kg}^{-1}$ (basal) plus FYM (5 mg Zn kg-1) treatment (Table 9).

All the two way interactions were found to be non-significant. By and large, level of DTPAextractable $\mathrm{Zn}$ was higher in all the soils after the harvest of rice than that after harvest of wheat (Fig 1). Similar trend was also reported by Mishra et al., (2009). In general, positive effect of applied $\mathrm{Zn}$ on subsequent crops in the rotation may last over variable periods (Brennan 2001).

In the absence of repeated $\mathrm{Zn}$ applications, soil Zn levels decreased gradually with successive cropping (Abid et al., 2013). Increase in the residual $\mathrm{Zn}$ content could be due to conversion of applied $\mathrm{Zn}$ of labile into non-labile forms.

In our study, the DTPA-extractable Zn was found higher in $5 \mathrm{mg} \mathrm{Zn} \mathrm{kg}$ (basal) plus FYM (5 mg Zn kg-1) as compared to other treatments which implied that FYM is capable of keeping applied $\mathrm{Zn}$ into more labile pool that becomes available to next crop. Therefore, $\mathrm{Zn}$ fertilizer requirement for second crop in cropping system be decided based on $\mathrm{Zn}$ fertilization history of previous crop.

Effectiveness of residual $\mathrm{Zn}$ in alleviating its deficiency in human by consuming wheat grown on residual $\mathbf{Z n}$

In the present investigation, daily dietary intake of $\mathrm{Zn}$ through consumption of $200 \mathrm{~g}$ of wheat was only $5.92 \mathrm{mg}$ day $^{-1}$ without previous $\mathrm{Zn}$ application and $6.61 \mathrm{mg} \mathrm{day}^{-1}$ with previous application through fertilizer and FYM.

There is an increase in $\mathrm{Zn}$ intake through wheat consumption by human due to residual $\mathrm{Zn}$. This increment would be higher if wheat is grown on a highly deficient soil as compared with $\mathrm{Zn}$-treated soil.

The current study showed that the $\mathrm{Zn}$ supplied through $\mathrm{ZnSO}_{4}$ has maintained a residual effect on to the next crop in addition to enhancing soil fertility and this residual $\mathrm{Zn}$ was effective in enhancing grain $\mathrm{Zn}$ concentrations in wheat in rice-wheat cropping system.

Based on the grain $\mathrm{Zn}$ concentrations, residual effect of $\mathrm{Zn}$ was more in CRIDA soils in which previously $\mathrm{Zn}$ was applied through zinc sulphate and FYM (Basal).

This residual effect can meet daily dietary intake of $\mathrm{Zn}$ by $6.61 \mathrm{mg} \mathrm{day}{ }^{-1}$ in humans, by consumption of $200 \mathrm{~g}$ of wheat grain. Although these are far below than daily recommended $\mathrm{Zn}$ intake, such increases will undoubtedly help in alleviating the widespread Zn deficiency in Indian population.

\section{Acknowledgements}

The support of Indian Agricultural Research Institute (IARI) and Council of Scientific and Industrial Research (CSIR) for providing all 
the facilities necessary for this study and stipend to the first author is gratefully acknowledged. The authors do not have any conflict of interest as part of the submission process.

\section{References}

Abid, M., Ahmed, N., Qayyum, M.F., Shaaban, M. and Rashid, A. 2013. Residual and cumulative effect of fertilizer zinc applied in wheat-cotton production system in an irrigated Aridisol. Plant Soil Environ., 59:505510.

Brennan, R.F. 2001. Residual value of zinc fertiliser for production of wheat. Aust $J$ Exp Agric., 41:541-547. doi: 10.1071/EA00139.

Brennan, R.F. and Bolland, M.D.A. 2007. Estimating the long-term residual value of zinc oxide for growing wheat in a sandy duplex soil. Aust J Agric Res., 58:57-65.

Cakmak, I. 2008. Enrichment of cereal grains with zinc: Agronomic or genetic biofortification. Plant Soil., 302:1-17.

Cakmak, I., Torun, B., Erenoglu, B., Kalayci, M., Yilmaz, A., Ekiz, H. and Braun, H.J. 2004. Zinc deficiency in soils and plants in Turkey and plant mechanisms involved in zinc efficiency. Plant Soil., 180:165-172.

Cakmak, I., Kalayci, M., Ekiz, H., Braun, H.J., Kilinc, Y. and Yilmaz, A. 1999. Zinc deficiency as a practical problem in plant and human nutrition in Turkey: A NATO-science for stability project. Field Crops. Res., 60:175-188.

Deshpande, J.D., Joshi, M.M. and Giri, P.A. 2013. Zinc: The trace element of major importance in human nutrition and health. Int J Med Sci Public Health., 2:1-6.

Dhaliwal, M.K., Dhaliwal, S.S., Thind, H.S. and Gupta, R.K. 2014. Long term effect of manure and fertilizers on concentration and uptake of $\mathrm{Zn}, \mathrm{Cu}, \mathrm{Fe}$ and $\mathrm{Mn}$ in rice and wheat grains under rice-wheat system. Int J Sci Environ., 3:1592-1601.

Gomez, K.A. and Gomez, A.A. 1984. Statistical procedures in agricultural research, New York, Chichester, etc. Wiley, Second edition, pp.680.

Grzetic, I., Rabia, H. and Ghariani, A. 2008. Potential health risk assessment for soil heavy metal contamination in the central zone of Belgrade (Serbia). $J$ Serb Chem Soc., 73:923-934.

Hague, Nnadi, L.A. and Mohammed Saleen, M.A. 2008. Phosphorus management with special reference to foreign legumes in Sub-Saharan Africa. Soils and Plant Nutrition Section ILCA, Addis Ababa, Ethiopia.

Hopkins, B. and Elisworth, J. 2003. Idaho Potato Commission Research and Extension Progress Report University of Idaho pp.76.

Hussain, F. 2004. Soil fertility monitoring and management in rice-wheat system. Annual Report, 2003-04 of the Agriculture Linkages Program Project at Land Resources Research Program, National Agricultural Research Centre Islamabad, Pakistan.

Jackson, M.L. 1973. In: Soil Chemical Analysis. Prentice Hall of India Pvt Ltd. New Delhi.

Jat, S.L., Shivay, Y.S., Kumar, D., Parihar, C.M. and Singh, A.K. 2012. Residual effects of dual-purpose summer legumes and zinc fertilization on succeeding wheat in aromatic hybrid rice-wheat cropping system. $J$ Wheat Res., 4(2).

Khan, R., Gurmani, A.R., Khan, M.S. and Gurmani, A.H. 2007. Effect of zinc application on rice yield under wheat rice system. Pak J Biol Sci., 10(2):235239. 
Khan, R., Gurmani, A.R., Khan, M.S. and Gurmani, A.H. 2009. Residual, direct and cumulative effect of zinc application on wheat and rice yield under rice-wheat system. Soil Environ., 28(1):24-28.

Kulhare, P.S., Sharma, G.D., Sharma, B.L. and Khamparia, R.S. 2014. Direct and residual effect of FYM and zinc uptake and available $\mathrm{Zn}$ in rice-wheat sequence on a vertisol. Indian Soc Soil Sci., 62:391-396.

Kumar, V., Kumar, A., Singh, S.K., Tripathi, S.K., Kumar, D, et al., 2016. Zinc Deficiency and Its Effect on the Brain: An Update. Int J Mol Genet Gene Therapy., 1(1): doi http://dx.doi.org/ 10.16966/2471- 4968.105

Lindsay, W.L. 1972. Zinc in soil and plant nutrition. Adv Agron., 24:147-186.

Lindsay, W.L., and Norvell, W.A. 1978. Development of a DTPA soil test for zinc, iron, manganese and copper. Soil Sci. Soc. Am. J. 42:421-428

Lyons, G., Ortiz, M.I., Stangoulis, J. and Graham, R. 2005. Selenium concentration in wheat grain: Is there sufficient genotypic variation to use in breeding? Plant Soil., 269:369-380.

Mahajan, A. and Gupta, R.D. 2009. RiceWheat cropping system. In: Integrated Nutrient Management (INM) in a sustainable rice-wheat cropping system. Springer, Nether lands. pp: 109-117. DOI: 10.1007/978-1-40209875-8_5

Mishra, P., Singh, R., Srivastava, P.C. and Ram, B. 2009. Effect of continuous cropping and fertilization on zinc fractions and their contribution to plant uptake under rice-wheat system. Indian Soc Soil Sci., 57:167-171.

Morgonuov, A., Gómez-Becerra, H.F., Abugalieva, A., Dzhunusova, M., Yessimbekova, M., Muminjanov, H., Zelenskiy, Y., Ozturk, L. and Cakmak,
I. 2007. Iron and zinc grain density in common wheat grown in Central Asia. Euphytica., 155:193-203.

Norton, R. 2012. Wheat grain nutrient concentrations for south-eastern Australia. In: Capturing opportunities and overcoming obstacles in Australian agronomy, ed. I. Yunusa. Proceedings of $16^{\text {th }}$ Australian Agronomy Conference, Armidale, NSW.

Ozturk, L., Yazici, M.A., Yucel, C., Torun, A., Cekic, C., Bagci, A., Ozkan, H., Braun, H.J., Sayers, Z. and Cakmak I. 2006. Concentration and localization of zinc during seed development and germination in wheat. Plan Physiol., 28:144-152.

Rashid, A. 2005. Establishment and management of micronutrients deficiencies in soils of Pakistan: A review. Soil Environ., 24(1):1-22.

Rattan, R.K., Datta, S.P. and Katyal, J.C. 2008. Micronutrient management: research achievements and future challenges. Indian $J$ Fertilisers., 4:103-106, 109-112 and 115-118.

Saharawat, Y.S., Bhagat Singh, R.K., Malik, J.K.,Ladha, M., Gathala, M.L., Jat, and Kumar, V. 2010. Evaluation of alternative tillage and crop establishment methods in a rice-wheat rotation in north-western IGP. Field Crops Res. 116:260-267. doi:10.1016/j.fcr.2010.01.003

Soleimani, R. 2012. Cumulative and residual effects of zinc sulfate on grain yield, zinc, iron, and copper concentration in corn and wheat. J Plant Nutr., 35:8592.

Soltangheisi, A., Rahman, Z.A., Ishak, C.F., Musa, H.M. and Zakikhani, H. 2014. Interaction effects of phosphorus and zinc on their uptake and ${ }^{32} \mathrm{P}$ absorption and translocation in sweet corn (Zea mays var. saccharata) grown in a 
tropical soil. Asian $J$ Plant Sci., 13:129-135.

Srinivasarao, Ch., Wani, S.P., Sahrawat, K.L., Rego, T.J. and Pardhasaradhi, G. 2008.

Zinc, boron and sulphur deficiencies are holding back the potential of rainfed crops in semi-arid India: Experiences from participatory watershed management. Int $J$ Plant Prod., 2:89-99.

Zhao, F.J., Su, Y.H., Dunham, S.J., Rakszegi,
M., Bedo, Z., McGrath, S.P. and Shewry, P.R. 2009. Variation in mineral micronutrient concentrations in grain of wheat lines of diverse origin. J Cereal Sci., 49:290-295.

Zhu, Y.G., Smith, S.E. and Smith, F.A. 2001. Zinc (Zn)-phosphorus (P) interactions in two cultivars of spring wheat (Triticum aestivum $\mathrm{L}$.) differing in $\mathrm{P}$ uptake efficiency. Ann Bot., 88:941945.

\section{How to cite this article:}

Girija Veni, V., S. P. Datta, R. K. Rattan, M. C. Meena and Sharma, K. L. 2019. Residual Effect of Zn Fertilization on Wheat (Triticum aestivum L.) Grown in Soils with Divergent Characteristics. Int.J.Curr.Microbiol.App.Sci. 8(10): 69-81. doi: https://doi.org/10.20546/ijcmas.2019.810.008 